\title{
Bone-Targeted Therapy in Metastatic Breast Cancer - All Well-Established Knowledge?
}

\author{
Simon P. Gampenrieder Gabriel Rinnerthaler Richard Greil \\ IIIrd Medical Department with Hematology, Medical Oncology, Hemostaseology, Infectious Diseases and Rheumatology, Oncologic Center, \\ Salzburg Cancer Research Institute (SCRI) with Laboratory of Immunological and Molecular Cancer Research (LIMCR) and Center for \\ Clinical Cancer and Immunology Trials (CCCIT), Paracelsus Medical University Salzburg, Salzburg, Austria
}

\section{Keywords}

Metastatic breast cancer - Bone metastasis .

Bone-targeted therapy · Bisphosphonates · Denosumab

\section{Summary}

Bone-targeted therapies like bisphosphonates (zoledronic acid or pamidronate) or denosumab are recommended in all patients with metastatic breast cancer and bone metastases, whether they are symptomatic or not. The choice between these 2 different agents, however, remains open. In this review, we critically discuss the emerging evidence for direct anti-tumor activity of bonetargeting agents, the utility of bone turnover markers for treatment decision and efficacy prediction, as well as the safety and financial aspects of bisphosphonates and denosumab. Furthermore, we provide a possible therapeutic algorithm, and present new pharmacologic agents which are being investigated for the treatment of metastatic bone disease.

\section{Introduction}

Accelerated bone loss is a frequent problem in patients with breast cancer. Besides treatment-induced bone loss due to endocrine therapies, approximately $70 \%$ of patients with advanced disease experience bone metastases [1], which can lead to so-called skeletal-related events (SREs) defined as pathological fractures, hypercalcemia, spinal cord compression, and the need for surgical intervention and/or radiation therapy. SREs are associated with shortened survival, deterioration of quality of life, and significant medical care costs [2-
4]. Bisphosphonates (BPs) are the most frequently used pharmacologic agents to prevent SREs and treatment-induced bone loss in patients with metastatic breast cancer $[5,6]$. Better understanding of bone remodeling led to the development of denosumab, a fully human monoclonal antibody against receptor activator of NFKB ligand (RANKL). Denosumab shows even higher efficacy in the prevention of SREs and treatment-induced bone loss than BPs [7, 8]. Despite the beneficial effects of BPs and RANKL-targeting agents, these agents are both associated with significant side effects, and the treatment of bone resorption and its deleterious clinical consequences in metastatic breast cancer still require further improvement. In this review, we critically discuss the emerging evidence for direct anti-tumor activity of bone-targeting agents, the utility of bone turnover markers for treatment decision and efficacy prediction, as well as the safety and financial aspects of BPs and denosumab. Furthermore, we present new pharmacologic agents which are being investigated for the treatment of metastatic bone disease.

\section{Bone-Targeted Effects of Established Antiresorptive Agents}

\section{Bisphosphonates}

\section{Mode of Action}

BPs are synthetic analogues of naturally occurring pyrophosphates, and based on their activity and chemical structure they are chronologically classified into 3 generations (table 1 ). BPs form bonds with crystal surfaces and inhibit hydroxyapatite crystal dissolution in bone tissue. In addition to this physicochemical stabilization of the bone structure, BPs are inter-

\section{KARGER}

Fax +497614520714

Information@Karger.com

www.karger.com
(ㄷ) 2014 S. Karger GmbH, Freiburg

$1661-3791 / 14 / 0095-0323 \$ 39.50 / 0$

Accessible online at:

www.karger.com/brc
Richard Greil, MD

IIIrd Medical Department

Salzburg Cancer Research Institute (SCRI)

Paracelsus Medical University Salzburg

Müllner Hauptstraße 48, 5020 Salzburg, Austria

r.greil@salk.at 
Table 1. The 3 generations of bisphosphonates

\begin{tabular}{lll}
\hline 1st Generation & 2nd Generation & 3rd Generation \\
\hline clodronate & alendronate & zoledronat \\
etidronate & ibandronate & minodronate \\
& pamidronate & risedronate \\
\hline
\end{tabular}

nalized by endocytosis from osteoclasts and metabolically incorporated into nonhydrolyzable analogues of adenosine triphosphate (ATP). These metabolites accumulate within osteoclasts, inhibit their absorption capacity, and induce apoptosis by inhibiting ATP-dependent enzymes. Nitrogencontaining BPs (N-BPs), second- and third-generation BPs, furthermore inhibit farnesyl diphosphate (FPP) synthase, a key enzyme of the mevalonate pathway. This capacity makes zoledronic acid (ZA) in some preclinical experiments up to 10,000-fold more potent than the first generation BP clodronate. Loss of FPP synthesis and its downstream metabolites prevents posttranslational modifications of small GTPases such as Ras, Rab, Rho, and Rac. These crucial signaling proteins regulate a variety of cell processes important for osteoclast function. Furthermore, disruption of the mevalonate pathway leads to an accumulation of isopentenyl pyrophosphate (IPP) in osteoclasts, which is converted to a cytotoxic ATP analogue called ApppI.

\section{Clinical Activity}

Several phase III trials demonstrated the ability of BPs to prevent and delay SREs in women with advanced breast cancer and clinically evident bone metastases [9-13]. A recent meta-analysis summarized 9 trials including 2,806 breast cancer patients comparing BPs with placebo or no BP, showing that BPs reduced the SRE risk by $15 \%$ (relative risk (RR) $0.85,95 \%$ confidence interval $(\mathrm{CI}) 0.77-0.94 ; \mathrm{p}=0.001$ ) [14]. In addition, BPs significantly reduced bone pain in 6 out of 11 studies, and improved global quality of life in 2 out of 5 studies; however, no effect on survival was observed in breast cancer patients with bone metastases [14]. As expected from preclinical studies [15], intravenous ZA showed the highest efficacy in reducing the risk of skeletal complications when compared to other BPs [16-20], representing therefore the most commonly used drug in oncological settings. The OPTIMIZE-2 trial, a phase III trial presented at the ASCO annual meeting 2014, demonstrated that after 1 year of treatment the dosing frequency of ZA can be reduced to 1 administration every 12 weeks without compromising effectiveness in women with breast cancer and bone metastases. The difference in SRE rate between the 2 arms was $1.2 \%(p=0.724)$, and the frequency of adverse events (AEs) was lower in the 12-week arm; however, this was not statistically significant (renal AEs 7.9 vs. $9.6 \%$, and osteonecrosis of the jaw (ONJ) 0 vs. $1.0 \%$ ) [21].

\section{Denosumab}

\section{Mode of Action}

Denosumab is a fully humanized $\mathrm{IgG}_{2}$ monoclonal antibody against RANKL. RANKL is a member of the tumor necrosis factor (TNF) superfamily, and is expressed on the surface of osteoblasts. Soluble RANKL is released into the bone microenvironment where it binds to and activates its receptor RANK on immature osteoclasts, acting as a key factor for osteoclast differentiation and activation. The expression level of RANKL is influenced by different hormones and cytokines such as macrophage-colony stimulating factor, TNF, prostaglandins (e.g. PGE2), steroids, parathyroid hormone (PTH), PTH-related protein (PTHrP), and interleukins (IL)-1, -6, -8 and -11 [22]. In bone metastases, these and other factors like macrophage inflammatory protein (MIP) $1 \alpha$ are secreted by tumor cells as well, leading to increased osteoclast activity. Furthermore, tumor cells excrete factors like Dickkopf-1 (DKK-1) and activin A that inhibit osteoblast differentiation. On the other hand, bone resorption releases growth factors (transforming growth factor $\beta$, insulin-like growth factors, fibroblast growth factors, and platelet-derived growth factor) from the bone matrix that stimulate tumor growth [23]. The monoclonal antibody denosumab binds RANKL and prevents bone resorption by inhibiting both mature osteoclast function and osteoclast differentiation, thereby interrupting this vicious cycle of bone destruction [7].

\section{Clinical Activity}

Three double-blind, phase III, registration trials with identical study design compared subcutaneous denosumab (120 mg, every 4 weeks) with intravenous ZA (4 mg adjusted for creatinine clearance, every 4 weeks) in patients with bone metastases secondary to breast cancer $(n=2,046)$, castration-resistant prostate cancer $(\mathrm{n}=1,901)$, and other tumors including nonsmall cell lung cancer $(\mathrm{n}=702)$, myeloma $(\mathrm{n}=180)$ and other types of solid tumors $(\mathrm{n}=894)$ [24-26]. In breast cancer patients, denosumab was superior to ZA in delaying time to first SRE, which represented the primary study endpoint. In addition, the secondary endpoint, time to first and subsequent SREs, was favoring denosumab [25]. Similar results were reported in patients with hormone-refractory prostate cancer [26]. In patients with bone metastases secondary to advancedstage solid tumors and myeloma, however, denosumab failed to demonstrate superiority over BPs [24]. When excluding the myeloma cohort (representing 10\% of the study population), an ad hoc analysis showed finally that denosumab was superior also in the solid tumor subset of this study [27] (table 2). The overlapping design of the 3 trials allowed a preplanned, integrated analysis of safety and efficacy data [8]. Overall, denosumab was superior to ZA in delaying time to first on-study SRE by a median of 8.2 months and reducing the risk of a first SRE by $17 \%$ (hazard ratio (HR) $0.83,95 \%$ CI $0.76-0.90$; p $<0.001)$. This superiority was consistent in various patient sub- 
Table 2. Phase

III trials comparing zoledronic acid $(4$ mg every 4 weeks intravenously) with denosumab $(120$ mg every 4 weeks subcutaneously)

\begin{tabular}{|c|c|c|c|}
\hline Author, year [ref.] & Patients, $\mathrm{n}$ & Population & Results (primary endpoint) \\
\hline Stopeck et al., 2010 [25] & 2,046 & breast cancer & $\begin{array}{l}\text { delayed time to first on-study SRE } \\
\text { (HR } 0.82, p<0.001 \text { non-inferiority, } \\
p=0.01 \text { superiority) }\end{array}$ \\
\hline Fizazi et al., 2011 [26] & 1,901 & prostate cancer & $\begin{array}{l}\text { delayed time to first on-study SRE } \\
\text { (HR } 0.82, p=0.0002 \text { non-inferiority, } \\
p=0.008 \text { superiority) }\end{array}$ \\
\hline Henry et al., 2011 [24] & 1,776 & $\begin{array}{l}\text { solid tumors (except breast } \\
\text { or prostate) and multiple } \\
\text { myeloma }\end{array}$ & $\begin{array}{l}\text { delayed time to first on-study SRE } \\
\text { (HR } 0.84, p=0.0007 \text { non-inferiority, } \\
p=\text { n.s. superiority) }\end{array}$ \\
\hline Henry et al., 2014 [27] & 1,597 & $\begin{array}{l}\text { solid tumors (except breast } \\
\text { or prostate) with multiple } \\
\text { myeloma excluded }\end{array}$ & $\begin{array}{l}\text { delayed time to first on-study SRE } \\
\text { (HR } 0.81, p=0.017 \text { superiority) }\end{array}$ \\
\hline
\end{tabular}

SRE = Skeletal-related events; HR = hazard ratio; n.s. = not significant. groups (prior SRE status, age). Denosumab was also superior to $\mathrm{ZA}$ in reducing the risk of multiple SRE by $18 \%$ (RR 0.82 , $95 \%$ CI $0.75-0.89 ; \mathrm{p}<0.001$ ) [8]. Based on the clinical activity, denosumab is approved not only for treatment of osteoporosis in postmenopausal women with high risk of fracture but also for bone loss in patients with cancer and bone metastases.

\section{Markers of Bone Turnover as Surrogate Endpoints}

Biochemical markers of bone formation and resorption, termed as bone turnover markers, are reflecting tumorinduced changes of bone metabolism. One of the most intensively investigated markers is urine amino-terminal crosslinked telopeptide of collagen type I (uNTx). Patients with high or moderate levels of uNTx have a 2-fold increased risk of skeletal complications and disease progression compared to patients with low uNTx levels $(\mathrm{p}<0.001)$ despite treatment with ZA $(n=1,462)$ or pamidronate $(n=362)$ [28]. Furthermore, persistently elevated uNtx after 3 months of ZA correlates with worse overall survival (OS) [29].

In a phase II trial conducted in patients with bone metastases caused by various tumor types, only patients with uNTx levels above the normal range ( $>50 \mathrm{nmol} / \mathrm{l} / \mathrm{mM}$ creatinine) despite ongoing intravenous BP treatment for at least 8 weeks were included [30]. Patients were randomly assigned to continue BPs or receive subcutaneous denosumab (180 mg every 4 weeks or $180 \mathrm{mg}$ every 12 weeks) for 25 weeks. A normalization of uNTx levels at week 13, the primary endpoint of this study, was achieved by $71 \%$ of patients in the denosumab arms, compared with $29 \%$ of patients in the BP arm $(\mathrm{p}<0.001)$ translating into lower SRE rates during the treatment phase in denosumab-treated patients. Additionally, in a retrospective analysis of the ZA phase III database, a normalization of initially elevated uNTx levels correlated with improved OS [27].

Other bone turnover markers under investigation are serum C-telopeptide ( $\mathrm{sCTx}$ ), aminoterminal propeptide type-1 procollagen (P1NP), osteocalcin, bone-specific alkaline phosphatase (BSAP), and tartrate-resistant acid phosphatase $5 b$ (TRAP-5b).

Since bone lesions are generally considered non-measurable according to RECIST 1.1 (Response Evaluation Criteria in Solid Tumors version 1.1), the assessment of therapy response in the case of bone-only disease is frequently difficult. There therefore exists a great clinical need for bone turnover markers corresponding to treatment response, especially if tumor markers like CA15-3 are not elevated.

\section{Antitumor Effects of Bone-Targeted Agents: Preclinical Evidence}

\section{Bisphosphonates}

There is growing preclinical evidence that the new generation of N-BPs harbor antitumor activity in addition to their effect on bone mineral density, while for denosumab similar data are missing.

BPs interact with macrophages, endothelial cells, and tumor cells, and stimulate the cytotoxicity of $\gamma \delta$ T-lymphocytes, a subset of human $\mathrm{T}$ cells that exhibits anticancer activity [31]. In a variety of cancer cell lines, BPs have demonstrated the ability to induce apoptosis as well as the capacity to decrease tumor cell adhesion, migration, and invasion [32].

Inhibition of the FPP synthetase seems to be the most important underlying antitumor mechanism of N-BPs. Disruption of the mevalonate pathway not only prevents posttranslational modifications (prenylation) of the small GTPases Ras, Rab, Rho, and Rac in tumor cells, but also leads to intracellular accumulation of IPP [33], mediates immuno-controlling effects on $\gamma \delta \mathrm{T}$-lymphocytes, and promotes antiangiogenic activity [34]. BPs also directly influence angiogenesis by degradation of hypoxia-inducible factor (HIF)- $1 \alpha$, leading to a decrease in vascular endothelial growth factor secretion in vitro [35].

Moreover, ZA inhibits tumor proliferation and migration by affecting the interaction between bone marrow-derived 


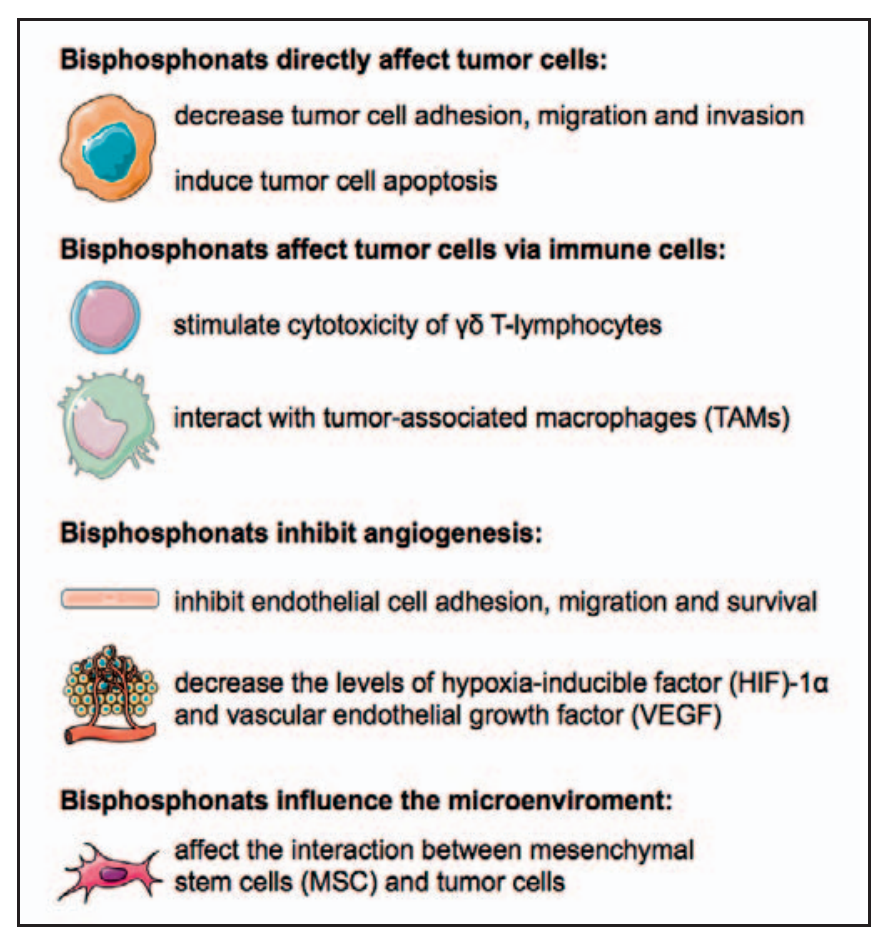

Fig. 1. Potential mechanisms of anti-tumor activity of bisphosphonates (drawings generated using Servier Medical Art; www.servier.com).

mesenchymal stem cells (MSCs) and breast cancer cells in vitro [36]. By affecting the recruitment of MSCs by primary tumors, ZA might influence an important mechanism of tumor progression. In addition, ZA sensitizes endothelial cells to TNF-induced (caspase-independent) apoptosis through inhibition of the FAK-PKB/Akt pathway [31] (fig. 1).

\section{Denosumab}

Whether denosumab harbors anticancer activity as well is largely unknown. RANKL and RANK are known to play an important role in mammary gland development [37], and studies in mouse models have shown that the RANKLRANK pathway is directly involved in mammary tumorigenesis $[38,39]$. In addition, RANKL production by tumor-infiltrating regulatory $\mathrm{T}$ cells promoted the development of pulmonary metastases in a RANK-positive breast cancer model [39]. Recent microarray analysis in human breast cancer biopsies showed that low RANK messenger RNA (mRNA) levels correlated with longer OS and disease-free survival, and, interestingly, a relatively increased expression of RANK mRNA was found in the basal-like breast cancer subtype when compared with non-basal-type tumors [40].

Data from a preclinical study in breast cancer cell lines and umbilical vein endothelial cells (HUVECs) as well as in vivo vascularization models supported the potential antiangiogenic and anticancer activity of ZA in vitro and in vivo but failed to demonstrate a similar effect of denosumab [41]. Additional studies are clearly warranted to elucidate the potential anticancer activity of denosumab.

\section{Antitumor Effects of Bone-Targeted Agents: Clinical Evidence}

\section{Bisphosphonates}

Clinical data supporting an antitumor effect of BPs are primarily derived from cancer prevention studies and adjuvant trials. Both the Women's Health Initiative Observational Study and the Breast Cancer in Northern Israel Study showed a significant reduction in the risk for breast cancer in women who received BPs (32 and $28 \%$ RR reduction, respectively) $[42,43]$. Furthermore, it has been shown that ZA given as a monthly dose in combination with chemotherapy reduced the number of disseminated tumor cells in the bone marrow of early breast cancer patients more efficiently than chemotherapy alone [44-47]. This effect might explain the results of the recently presented meta-analysis of 22 trials including almost 18,000 early breast cancer patients, where the addition of BPs to standard adjuvant treatment significantly reduced the risk for distant recurrence and breast cancer death [48].

In the metastatic setting, ZA seems to prolong OS in patient with metastatic bone disease from different solid tumors only in patients with high uNTx levels at baseline, but these data have to be interpreted with caution because of their exploratory nature [49]. A meta-analysis of 9 trials including 2,806 metastatic breast cancer patients comparing BPs with placebo or control revealed no effect on OS in the overall population [14], while in lung cancer or prostate cancer such an effect was observed $[50,51]$.

\section{Denosumab}

Denosumab significantly increased bone metastases-free survival compared with placebo in a phase III trial including 1,432 men with non-metastatic castration-resistant prostate cancer at high risk of bone metastasis (prostate-specific antigen $(\mathrm{PSA}) \geq 8.0 \mu \mathrm{g} / 1$ or PSA doubling time $\leq 10.0$ months, or both) by a median of 4.2 months (HR $0.85,95 \%$ CI $0.73-0.98$; $\mathrm{p}=0.028)$. However, no influence on OS was seen when compared to placebo (HR 1.01, 95\% CI 0.85-1.20; $\mathrm{p}=0.91$ ) [52]. A meta-analysis summarizing trials comparing denosumab with ZA in patients with bone metastases secondary to breast cancer, prostate cancer, other solid tumors, or multiple myeloma neither showed any difference in OS (HR 0.99, 95\% CI $0.91-1.07 ; \mathrm{p}=0.71$ ) nor in disease progression (HR 1.02, 95\% CI $0.95-1.08 ; \mathrm{p}=0.63$ ) [8].

\section{Safety of Bone-Targeted Therapies}

\section{Bisphosphonates}

The most frequent side effects from intravenous BPs are fever and myalgias (acute-phase reactions, APR), which may occur in up to $55 \%$ of cases mostly within $24 \mathrm{~h}$ of the first infusion. Antipyretic and anti-inflammatory agents usually provide easy relief [53]. Importantly, not all BPs induce APR to the 
same extent (ZA more than the others). Pathophysiologically, APR is attributed to a transient release of pro-inflammatory cytokines (mostly TNF- $\alpha$, IL-6, interferon- $\gamma$ ) from $\gamma \delta$ T-lymphocytes, which are activated in response to BPs.

Electrolyte alterations, including hypophosphatemia, hypocalcemia, and hypo- or hypermagnesemia, are other known disturbances associated with intravenous BPs. Therefore, prophylactic substitution of calcium and vitamin D are recommended [53].

Renal toxicity is a major issue with intravenous N-BPs. An increase in creatinine levels from baseline is reported in about $10 \%$ of patients receiving BP treatment, but the frequency varies depending on the specific $\mathrm{BP}$, the dose schedule, the duration of administration, and concomitant medications. Creatinine clearance should be measured prior to administration of intravenous BPs, and dose adjustments according to treatment guidelines as well as prolonged infusion times may help to reduce this problem [53].

$\mathrm{ONJ}$ is an infrequent $\mathrm{AE}$ reported in $1.3 \%$ of patients with bone metastases receiving ZA [8]. Risk factors for the development of this serious side effect are poor oral hygiene, history of dental extraction or use of dental appliance, preexisting dental or periodontal disease, usage of glucocorticosteroids and antiangiogenic agents, or radiation therapy [53, 54]. Therefore, before starting intravenous N-BP treatment, patients should have a dental examination, and surgical dental procedures, if required, should ideally be completed before initiation [55].

Oral BPs like ibandronate or clodronate may in addition provoke esophageal or gastric irritation and diarrhea [16].

\section{Denosumab}

Renal AEs and APR occur significantly less frequently with denosumab when compared with ZA [8]. Unlike BPs, denosumab is not excreted via the kidneys but is eliminated by intracellular catabolism in phagocytes, similar to the clearance mechanism of other therapeutic monoclonal antibodies, and can therefore be safely used in patients with chronic kidney disease [56, 57].

In the registration trials, hypocalcemia of any grade was 2-fold higher in the denosumab group compared to ZA, and severe hypercalcemia $(<1.75 \mathrm{mmol} / \mathrm{l})$ was reported in 3.1 versus $1.3 \%$ [8]. Several case reports of prolonged severe hypocalcemia, consistent with our own experience, are reported in the literature $[58,59]$. Elucidation of predictive factors for this high bone turnover state caused by denosumab is therefore greatly needed. Baseline calcium serum levels within the normal range, as recommended in the prescribing information, should be a mandatory prerequisite.

In a meta-analysis comparing denosumab with $\mathrm{ZA}$, ONJ occurred at a higher rate in the denosumab arm; however, the difference was not statistically significant (1.8 vs. $1.3 \%$; $\mathrm{p}=$ 0.13) [8]. In contrast to BPs, dental procedures are thought to be safe after a therapy pause of only 26 days (compared to 6 months with N-BPs) because of the much shorter half-life of denosumab.

A statistically increased risk of pancreatitis and serious infections, including endocarditis, erysipelas, and infectious arthritis, has been reported with this drug as well [53].

\section{Economic Considerations}

SREs are associated with relevant medical care costs. A retrospective study in metastatic breast cancer in the US showed that the average cost of treatment of an SRE was $\$ 13,940$ (95\% CI \$11,240-16,856) [2]. Although denosumab has demonstrated benefit over ZA in preventing or delaying SREs in different phase III trials, ZA is less expensive, and the higher efficacy of denosumab has to be weighed against the incremental costs. On the other hand, the ease of subcutaneous injection and the lack of a need to closely monitor creatinine levels have to be taken into account. The economic point of view becomes even more important since the economic pressure on healthcare systems all over the world is growing, and ZA became generic in the EU in November 2013.

\section{Future Perspectives}

\section{Targeting Signaling Pathways}

A number of new compounds are under investigation for the treatment of metastatic bone disease (fig. 2). Agents targeting the Src family kinases (e.g. dasatinib, saracatinib, KX2391, and bosutinib) showed promising preclinical and earlyphase clinical data. A recently presented phase II trial of dasatinib in combination with letrozol in metastatic breast cancer not only showed a reduced percentage of patients with a T-score $<-1.5$ but also a significantly longer progression-free survival (PFS) in the combination arm compared with letrozol alone [60]. A phase II trial investigating bosutinib, which inhibits the Src/Abl tyrosine kinase as well as many other kinases, showed promising antitumor activity as single agent as well; however, no consistent changes in the levels of bone turnover markers were seen [61].

Odanacatib (MK-0822), an inhibitor of cathepsin K, suppressed uNTx similarly to ZA after 4 weeks of treatment in women with breast cancer and bone metastases [62]. A phase III trial in metastatic prostate cancer, however, has been withdrawn prior to enrollment for administrative reasons (NCT00691899), and further development of odanacatib in the oncology field is on hold.

Exploratory analyses from the BOLERO-2 trial in metastatic breast cancer showed that the mTOR inhibitor everolimus may have beneficial effects on bone metabolism, potentially reducing bone resorption and contributing to a boneprotective effect [63]. 


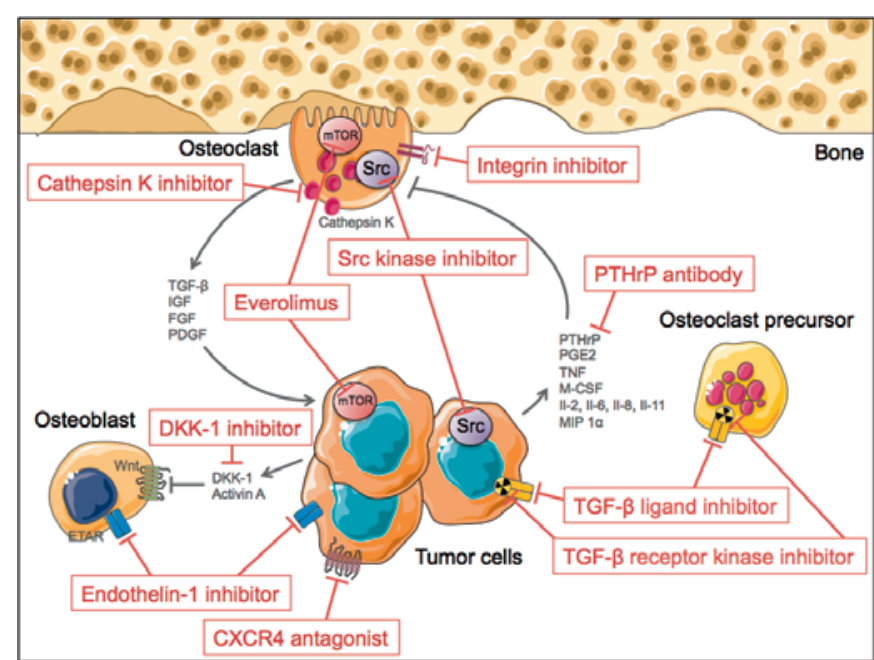

Fig. 2. Promising compounds under investigation for the treatment of metastatic bone disease (drawings generated using Servier Medical Art; www.servier.com).

Other promising targets like chemokine receptor 4 (CXCR4), parathyroid hormone-related peptide (PTHrP), transforming growth factor $\beta$ (TGF- $\beta$ ), DKK-1, integrins, or endothelin-1 are under investigation, but clinical data in breast cancer are still missing $[64,65]$.

\section{Radiopharmaceuticals}

Radium-223 (Xofigo ${ }^{\circledR}$, Bayer Healthcare, Leverkusen, Germany), a first-in-class alpha-emitting radiopharmaceutical, acts by delivering cytotoxic alpha-particle radiation to sites of bone metastasis. This highly targeted cytotoxic effect on bone metastases has led to the approval for the treatment of patients with castration-resistant prostate cancer and symptomatic bone metastases with no known visceral metastatic disease. A phase III trial in this population $(\mathrm{n}=921)$ showed that radium-223, compared with placebo, significantly improved median OS by 3.6 months (HR $0.70,95 \%$ CI $0.58-$ $0.83 ; \mathrm{p}<0.001$ ), significantly delayed the time to first SRE (HR $0.66,95 \%$ CI $0.52-0.83 ; \mathrm{p}<0.001$ ), and improved quality of life [66]. In breast cancer, only phase II data are available: 21 patients with advanced bone-dominant disease not being candidates for further endocrine therapy received radium-223 (50 kBq/kg intravenously) every 4 weeks for 4 cycles [67]. Therapy resulted in a significant reduction in uNTx and $\mathrm{BSAP}$, and the metabolic response rate (defined as $\geq 25 \%$ reduction in maximum standardized FDG uptake value from baseline in positron emission tomography/computed tomography) was $32 \%$ at week 9 and $42 \%$ at week 17 . Treatment was generally well-tolerated, and most AEs considered to be possibly or probably treatment-related were mild and reversible, with nausea, diarrhea, anorexia, vomiting, constipation, fatigue, and bone pain being the most frequently reported (mostly grade 1-2; grade 3 AEs occurred in only 5 patients). Grade 3 hematologic AEs were infrequent as well, with 1 case

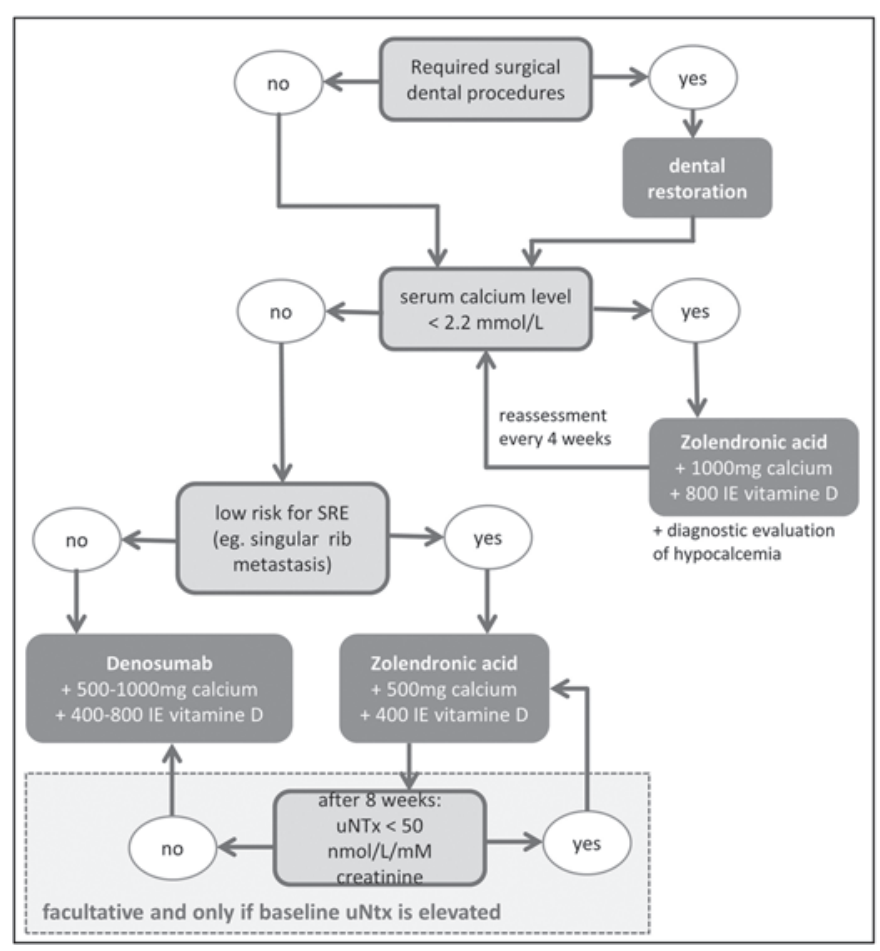

Fig. 3. Proposed treatment decision algorithm.

of anemia and 1 case of neutropenia [67]. Further studies with radium-223 in this patient population are being planned.

Other bone-seeking radiopharmaceuticals like phosphorus-32, strontium-89, rhenium-186, rhenium-188, and samarium-153 have shown promising results in terms of reducing pain from diffuse skeletal metastases [68], but further data are needed to assess their effect on disease progression.

\section{Conclusion - All Well-Established Knowledge?}

Bone-targeted therapies are of undisputed importance in the multimodal therapy of metastatic breast cancer involving the bone. Initiation of an N-BP (ZA or pamidronate) or denosumab is recommended in all patients with metastatic breast cancer and bone metastases, whether they are symptomatic or not [69]. The best choice between these 2 different agents, however, remains open. The guidelines from the American Society of Clinical Oncology (ASCO) state that there is insufficient evidence to demonstrate greater efficacy of one bone-modifying agent over another [70]. The greater efficacy, demonstrated in clinically relevant endpoints like time to first and subsequent SRE in a prospective doubleblind phase III trial [25], might be considered in favor of denosumab. The ease of subcutaneous injection and the lack of a need to closely monitor creatinine levels are further advantages. The lack of differences in PFS and OS as well as in the rate of ONJ, the significantly higher costs, and the higher rate of grade 3 hypocalcemia may, however, influence treatment 
choice in favor of BP, especially if the risk for an SRE is low. Another possible clinical strategy could be to reserve denosumab for patients whose uNTx levels failed to normalize after BP treatment. A possible algorithm for treatment choice is provided in figure 3 .

Many questions still remain. What is the optimal treatment duration? What is the role of newer bone-targeting agents? How should radiopharmaceuticals be included into the therapeutic algorithm? Ongoing studies will answer some of these questions, and future research efforts may provide additional encouraging treatment options for patients with metastatic bone disease.

\section{Disclosure Statement}

Employment or leadership position: none; consultant or advisory role: R. Greil for Roche, Celgene, Teva, Takeda, BMS, Novartis; stock ownership: none; speakers honoraria: R. Greil for Roche; travel grants: S.P. Gampenrieder from Roche, Amgen, GlaxoSmithKline, and G. Rinnerthaler from Roche, Amgen, Merck, Pfizer; research funding: R. Greil from Roche, Novartis, Amgen, Takeda, Teva, and S.P. Gampenrieder and G. Rinnerthaler from Roche.

\section{References}

$\checkmark 1$ Coleman RE: Metastatic bone disease: clinical features, pathophysiology and treatment strategies. Cancer Treat Rev 2001;27:165-176.

$\checkmark 2$ Delea T, McKiernan J, Brandman J, et al. Retrospective study of the effect of skeletal complications on total medical care costs in patients with bone metastases of breast cancer seen in typical clinical practice. J Support Oncol 2006;4:341-347.

3 Costa L, Badia X, Chow E, et al.: Impact of skeletal complications on patients' quality of life, mobility, and functional independence. Support Care Cancer 2008;16:879-889.

$\checkmark 4$ Norgaard M, Jensen AO, Jacobsen JB, et al. Skeletal related events, bone metastasis and surviva of prostate cancer: a population based cohort study in Denmark (1999 to 2007). J Urol 2010;184:162-167.

$\checkmark 5$ Coleman RE: Bisphosphonates in breast cancer. Ann Oncol 2005;16:687-695.

6 Russell RG: Bisphosphonates: the first 40 years. Bone 2011;49:2-19.

7 Brown JE, Coleman RE: Denosumab in patients with cancer-a surgical strike against the osteoclast. Nat Rev Clin Oncol 2012;9:110-118.

$>8$ Lipton A, Fizazi K, Stopeck AT, et al.: Superiority of denosumab to zoledronic acid for prevention of skeletal-related events: a combined analysis of 3 pivotal, randomised, phase 3 trials. Eur J Cancer 2012;48:3082-3092.

$\checkmark 9$ Body JJ, Diel IJ, Lichinitzer M, et al.: Oral ibandronate reduces the risk of skeletal complications in breast cancer patients with metastatic bone disease: results from two randomised, placebo-controlled phase iii studies. Br J Cancer 2004;90:1133-1137.

10 Kohno N, Aogi K, Minami H, et al.: Zoledronic acid significantly reduces skeletal complications compared with placebo in Japanese women with bone metastases from breast cancer: a randomized, placebo-controlled trial. J Clin Oncol 2005;23:3314-3321.

11 Lipton A, Theriault RL, Hortobagyi GN, et al.: Pamidronate prevents skeletal complications and is effective palliative treatment in women with breast carcinoma and osteolytic bone metastases: long term follow-up of two randomized, placebo-controlled trials. Cancer 2000;88:1082-1090.

12 Theriault RL, Lipton A, Hortobagyi GN, et al.: Pamidronate reduces skeletal morbidity in women with advanced breast cancer and lytic bone lesions: a randomized, placebo-controlled trial. Protocol 18 Aredia Breast Cancer Study Group. J Clin Oncol 1999;17:846-854.
13 Tripathy D, Lichinitzer M, Lazarev A, et al.: Oral ibandronate for the treatment of metastatic bone disease in breast cancer: efficacy and safety results from a randomized, double-blind, placebo-controlled trial. Ann Oncol 2004;15:743-750.

14 Wong MH, Stockler MR, Pavlakis N: Bisphosphonates and other bone agents for breast cancer. Cochrane Database Syst Reviews 2012;2:CD003474.

15 Green JR, Muller K, Jaeggi KA: Preclinical pharmacology of CGP 42'446, a new, potent, heterocyclic bisphosphonate compound. J Bone Miner Res 1994;9:745-751.

16 Barrett-Lee P, Casbard A, Abraham J, et al.: Oral ibandronic acid versus intravenous zoledronic acid in treatment of bone metastases from breast cancer: a randomised, open label, non-inferiority phase 3 trial. Lancet Oncol 2014;15:114-122.

17 Major P, Lortholary A, Hon J, et al.: Zoledronic acid is superior to pamidronate in the treatment of hypercalcemia of malignancy: a pooled analysis of two randomized, controlled clinical trials. J Clin Oncol 2001;19:558-567.

18 Rosen LS, Gordon D, Kaminski M, et al.: Longterm efficacy and safety of zoledronic acid compared with pamidronate disodium in the treatment of skeletal complications in patients with advanced multiple myeloma or breast carcinoma: a randomized, double-blind, multicenter, comparative trial. Cancer 2003;98:1735-1744.

19 Rosen LS, Gordon DH, Dugan W Jr, et al.: Zoledronic acid is superior to pamidronate for the treatment of bone metastases in breast carcinoma patients with at least one osteolytic lesion. Cancer 2004;100:36-43.

20 Coleman RE: Efficacy of zoledronic acid and pamidronate in breast cancer patients: a comparative analysis of randomized phase III trials. Am J Clin Oncol 2002;25:S25-31.

21 Hortobagyi GN, Lipton A, Chew HK, et al.: Efficacy and safety of continued zoledronic acid every 4 weeks versus every 12 weeks in women with bone metastases from breast cancer: results of the optimize-2 trial. J Clin Oncol 2014;32:5s

22 Dougall WC, Chaisson M: The RANK/RANKL/ OPG triad in cancer-induced bone diseases. Cancer Metastasis Rev 2006;25:541-549.

23 Coleman R, Gnant M, Morgan G, Clezardin P: Effects of bone-targeted agents on cancer progression and mortality. J Natl Cancer Inst 2012;104: 1059-1067.
24 Henry DH, Costa L, Goldwasser F, et al.: Randomized, double-blind study of denosumab versus zoledronic acid in the treatment of bone metastases in patients with advanced cancer (excluding breast and prostate cancer) or multiple myeloma. J Clin Oncol 2011;29:1125-1132.

25 Stopeck AT, Lipton A, Body JJ, et al.: Denosumab compared with zoledronic acid for the treatment of bone metastases in patients with advanced breast cancer: a randomized, double-blind study. J Clin Oncol 2010;28:5132-5139.

26 Fizazi K, Carducci M, Smith M, et al.: Denosumab versus zoledronic acid for treatment of bone metastases in men with castration-resistant prostate cancer: a randomised, double-blind study. Lancet 2011;377:813-822.

27 Henry D, Vadhan-Raj S, Hirsh V, et al.: Delaying skeletal-related events in a randomized phase 3 study of denosumab versus zoledronic acid in patients with advanced cancer: an analysis of data from patients with solid tumors. Support Care Cancer 2014;22:679-687.

28 Coleman RE, Major P, Lipton A, et al.: Predictive value of bone resorption and formation markers in cancer patients with bone metastases receiving the bisphosphonate zoledronic acid. J Clin Oncol 2005; 23:4925-4935.

29 Lipton A, Cook R, Saad F, et al.: Normalization of bone markers is associated with improved survival in patients with bone metastases from solid tumors and elevated bone resorption receiving zoledronic acid. Cancer 2008:113:193-201.

30 Fizazi K, Lipton A, Mariette X, et al.: Randomized phase ii trial of denosumab in patients with bone metastases from prostate cancer, breast cancer, or other neoplasms after intravenous bisphosphonates. J Clin Oncol 2009;27:1564-1571.

-31 Clezardin P: Bisphosphonates' antitumor activity: an unravelled side of a multifaceted drug class. Bone 2011;48:71-79.

32 Neville-Webbe HL, Gnant M, Coleman RE: Potential anticancer properties of bisphosphonates. Seminars in oncology 2010;37 Suppl 1:S53-65.

33 Stresing V, Fournier PG, Bellahcene A, et al.: Nitrogen-containing bisphosphonates can inhibit angiogenesis in vivo without the involvement of farnesyl pyrophosphate synthase. Bone 2011;48: 259-266.

34 Stresing V, Daubine F, Benzaid I, et al.: Bisphosphonates in cancer therapy. Cancer Lett 2007;257: $16-35$. 
-35 Tang X, Zhang Q, Shi S, et al.: Bisphosphonates suppress insulin-like growth factor 1-induced angiogenesis via the HIF-1alpha/VEGF signaling pathways in human breast cancer cells. Int J Cancer 2010;126:90-103.

36 Gallo M, De Luca A, Lamura L, Normanno N: Zoledronic acid blocks the interaction between mesenchymal stem cells and breast cancer cells: implications for adjuvant therapy of breast cancer. Ann Oncol 2012;23:597-604.

37 Fata JE, Kong YY, Li J, et al.: The osteoclast differentiation factor osteoprotegerin-ligand is essential for mammary gland development. Cell 2000; 103:41-50.

-38 Gonzalez-Suarez E, Jacob AP, Jones J, et al.: Rank ligand mediates progestin-induced mammary epithelial proliferation and carcinogenesis. Nature 2010;468:103-107.

-39 Tan W, Zhang W, Strasner A, et al.: Tumourinfiltrating regulatory $\mathrm{T}$ cells stimulate mammary cancer metastasis through RANKL-RANK signalling. Nature 2011;470:548-553.

40 Santini D, Schiavon G, Vincenzi B, et al.: Receptor activator of NF-kB (RANK) expression in primary tumors associates with bone metastasis occurrence in breast cancer patients. PloS One 2011;6:e19234.

41 Misso G, Porru M, Stoppacciaro A, et al.: Evaluation of the in vitro and in vivo antiangiogenic effects of denosumab and zoledronic acid. Cancer Biol Ther 2012;13:1491-1500.

42 Chlebowski RT, Chen Z, Cauley JA, et al.: Oral bisphosphonate use and breast cancer incidence in postmenopausal women. J Clin Oncol 2010;28: 3582-3590.

43 Rennert G, Pinchev M, Rennert HS: Use of bisphosphonates and risk of postmenopausal breast cancer. J Clin Oncol 2010;28:3577-3581.

44 Aft R, Naughton M, Trinkaus K, et al.: Effect of zoledronic acid on disseminated tumour cells in women with locally advanced breast cancer: an open label, randomised, phase 2 trial. Lancet Oncol 2010;11:421-428.

45 Rack B, Juckstock J, Genss EM, et al.: Effect of zoledronate on persisting isolated tumour cells in patients with early breast cancer. Anticancer Res 2010;30:1807-1813.

46 Solomayer EF, Gebauer G, Hirnle P, et al. Influence of zoledronic acid on disseminated tumor cells in primary breast cancer patients. Ann Oncol 2012;23:2271-2277.

47 Banys M, Solomayer EF, Gebauer G, et al.: Influence of zoledronic acid on disseminated tumor cells in bone marrow and survival: results of a prospective clinical trial. BMC Cancer 2013;13:480.
48 Coleman R, Gnant M, Paterson A, et al.: Effects of bisphosphonate treatment on recurrence and cause-specific mortality in women with early breast cancer: a meta-analysis of individual patient data from randomised trials. Cancer Res 2013;73(24 suppl):abstr S4-07.

49 Cook RJ, Hirsh V, Major PP, et al.: Meta-analysis of effects of zoledronic acid (ZOL) on survival in metastatic bone disease (MBD): survival in patients with high bone turnover. J Clin Oncol 2008; 26:abstr 20562

50 Dearnaley DP, Mason MD, Parmar MK, et al.: Adjuvant therapy with oral sodium clodronate in locally advanced and metastatic prostate cancer: long-term overall survival results from the MRC PR04 and PR05 randomised controlled trials. Lancet Oncol 2009;10:872-876.

51 Zarogoulidis K, Boutsikou E, Zarogoulidis P, et al.: The impact of zoledronic acid therapy in survival of lung cancer patients with bone metastasis. Int J Cancer 2009;125:1705-1709.

52 Smith MR, Saad F, Coleman R, et al.: Denosumab and bone-metastasis-free survival in men with castration-resistant prostate cancer: results of a phase 3, randomised, placebo-controlled trial. Lancet 2012;379:39-46.

53 Mortimer JE, Pal SK: Safety considerations for use of bone-targeted agents in patients with cancer. Semin Oncol 2010;37(suppl 1):S66-72.

54 Saad F, Brown JE, Van Poznak C, et al.: Incidence, risk factors, and outcomes of osteonecrosis of the jaw: ntegrated analysis from three blinded activecontrolled phase iii trials in cancer patients with bone metastases. Ann Oncol 2012;23:1341-1347.

55 Weitzman R, Sauter N, Eriksen EF, et al.: Critical review: updated recommendations for the prevention, diagnosis, and treatment of osteonecrosis of the jaw in cancer patients - May 2006. Crit Rev Oncol Hematol 2007;62:148-152.

56 Block GA, Bone HG, Fang L, et al.: A single-dose study of denosumab in patients with various degrees of renal impairment. J Bone Miner Res 2012; 27:1471-1479.

57 Wang W, Wang EQ, Balthasar JP: Monoclonal antibody pharmacokinetics and pharmacodynamics. Clin Pharmacol Ther 2008;84:548-558.

58 Kreutle V, Blum C, Meier C, et al.: Bisphosphonate induced hypocalcaemia - report of six cases and review of the literature. Swiss Med Wkly 2014;144: w13979.

59 Milat F, Goh S, Gani LU, et al.: Prolonged hypocalcemia following denosumab therapy in metastatic hormone refractory prostate cancer. Bone 2013;55:305-308.
60 Paul D, Vukelja SJ, Holmes FA, et al.: Letrozole plus dasatinib improves progression-free survival (PFS) in hormone receptor-positive, HER2negative postmenopausal metastatic breast cancer (MBC) patients receiving first-line aromatase inhibitor (AI) therapy. Cancer Res 2013;73(24 suppl):abstr S3-07.

61 Campone M, Bondarenko I, Brincat S, et al.: Phase II study of single-agent bosutinib, a Src/Abl tyrosine kinase inhibitor, in patients with locally advanced or metastatic breast cancer pretreated with chemotherapy. Ann Oncol 2012;23:610-617.

62 Jensen AB, Wynne C, Ramirez G, et al.: The cathepsin $\mathrm{K}$ inhibitor odanacatib suppresses bone resorption in women with breast cancer and established bone metastases: results of a 4-week, doubleblind, randomized, controlled trial. Clin Breast Cancer 2010;10:452-458.

63 Hadji P, Coleman R, Gnant M: Bone effects of mammalian target of rapamycin (mTOR) inhibition with everolimus. Crit Rev Oncol Hematol 2013;87:101-111.

64 Onishi T, Hayashi N, Theriault RL, et al.: Future directions of bone-targeted therapy for metastatic breast cancer. Nat Rev Clin Oncol 2010;7:641-651.

65 Deng X, He G, Liu J, et al.: Recent advances in bone-targeted therapies of metastatic prostate cancer. Cancer Treat Rev 2014;40:730-738.

-66 Parker C, Nilsson S, Heinrich D, et al.; ALSYMPCA Investigators: Alpha emitter radium-223 and survival in metastatic prostate cancer. N Engl J Med 2013;369:213-223.

67 Coleman R, Aksnes AK, Naume B, et al.: A phase IIa, nonrandomized study of radium-223 dichloride in advanced breast cancer patients with bone-dominant disease. Breast Cancer Res Treat 2014;145: 411-418.

68 Rubini G, Nicoletti A, Rubini D, Asabella AN Radiometabolic treatment of bone-metastasizing cancer: from 186rhenium to 223radium. Cancer Biother Radiopharm 2014;29:1-11.

69 Coleman R, Body JJ, Aapro M, et al.; on behalf of the EGWG: Bone health in cancer patients: ESMO clinical practice guidelines. Ann Oncol 2014;25 (suppl 3):iii124-iii137.

70 Van Poznak CH, Temin S, Yee GC, et al.; American Society of Clinical Oncology: American Society of Clinical Oncology executive summary of the clinical practice guideline update on the role of bone-modifying agents in metastatic breast cancer. J Clin Oncol 2011;29:1221-1227. 